\title{
BONNET PAIRS AND ISOTHERMIC SURFACES
}

\author{
GEORGE KAMBEROV, FRANZ PEDIT, AND ULRICH PINKALL
}

\section{INTRODUCTION}

A classical question in surface theory is which data are sufficient to describe a surface in space up to rigid motions. Bonnet suggested that mean curvature and metric should suffice to determine the surface generically. The local theory was developed by Bonnet [6], Cartan [4] and Chern [5] who showed the existence of various 1-parameter families of Bonnet surfaces, i.e., surfaces with the same induced metric and mean curvature. A comprehensive study of this problem and its relationship to the Painleve equations has recently been completed by Bobenko and Eitner [2]. On the other hand, Lawson and Tribuzy [9] have shown that for embedded compact surfaces there are at most two surfaces to a given metric and mean curvature. Moreover, uniqueness can be established under various global assumptions [8]. Up to date it is unknown whether such compact Bonnet pairs exist and if, how to construct them.

In this note we classify all Bonnet pairs on a simply connected domain. Our main intent was to apply what we call a quaternionic function theory to a concrete problem in differential geometry. In the first section we develop the necessary formulas and explain how usual Riemann surface theory can be viewed as a special case of our extended function theory. The ideas are simple: conformal immersions into quaternions or imaginary quaternions take the place of chart maps for a Riemann surface. Starting from a reference immersion we construct all conformal immersions of a given Riemann surface (up to translational periods) by spin transformations (Definition 2.1).With this viewpoint in mind we discuss in the second section how to construct all Bonnet pairs on a simply connected domain from isothermic surfaces and vice versa. Isothermic surfaces are solutions to a certain soliton equation [1] thus a simple dimension count tells us that we obtain Bonnet pairs which are not part of any of the families. The corresponcence between Bonnet pairs and isothermic surfaces is explicit and to each isothermic surface we obtain a 4-parameter family of Bonnet pairs.

\section{Surface THEORY REVISITED}

Let $M$ be a Riemann surface and $f: M \rightarrow \mathbb{R}^{3}$ an immersion. We always regard $\mathbb{R}^{4}=\mathbb{H}$ as quaternions and $\mathbb{R}^{3}=\operatorname{Im} \mathbb{H}$ as purely imaginary quaternions. Note that for $a, b \in \operatorname{Im} \mathbb{H}$ we have

$$
a b=-<a, b>+a \times b .
$$

There is a natural wedge product over $\mathbb{H}$-valued 1 -forms given by

$$
\alpha \wedge \beta(X, Y)=\alpha(X) \beta(Y)-\alpha(Y) \beta(X)
$$

First and second author were partially supported by NSF grant DMS-9205293. Third author was supported by a Five Colleges Distinguished Visiting Professorship and NSF grant DMS-9205293. 
which satisfies the obvious identities

$$
\begin{gathered}
\overline{\alpha \wedge \beta}=-\bar{\beta} \wedge \bar{\alpha} \\
\alpha \wedge h \beta=\alpha h \wedge \beta \\
\mathrm{d}(h \alpha)=d h \wedge \mathrm{d} \alpha+h \mathrm{~d} \alpha \\
\mathrm{d}(\alpha h)=\mathrm{d} \alpha h-\alpha \wedge d h
\end{gathered}
$$

for an $\mathbb{H}$-valued function $h: M \rightarrow \mathbb{H}$. Usually we will identify 2-forms on $M$ with their quadratic forms via

$$
\omega(X)=\omega(X, J X)
$$

where $\omega \in \Omega^{2}(M, \mathbb{H})$ and $J: T M \rightarrow T M$ is the complex structure of $M$. Thus the only objects appearing in our theory will be $\mathbb{H}$-valued functions on $M$ and $T M$. A key formula is

$$
\alpha \wedge \beta=\alpha(* \beta)-(* \alpha) \beta
$$

where $* \alpha=\alpha \circ J$ is minus the usual Hodge star operator. With this in place let us develop the necessary equations of surface theory as needed for our purposes.

Lemma 2.1. $f: M \rightarrow \mathbb{R}^{3}$ is a conformal immersion if and only if there exists $N: M \rightarrow \mathbb{H}$ such that

$$
* d f=N d f .
$$

If (2.3) holds then $N: M \rightarrow S^{2} \subset \mathrm{Im} \mathbb{H}$ is the (oriented) unit normal field (Gauss map) to $f$.

Proof. Since $*^{2}=-1$ and $d f$ is pointwise injective we have $N^{2}=-1$. This implies $|N|^{2}=1$ and thus $\bar{N}=-N$. Conjugating (2.3) gives $N d f=-d f N$ which says that $\langle N, d f\rangle=0$. Thus $N: M \rightarrow S^{2} \subset \operatorname{Im} \mathbb{H}$ is a unit normal field to $f$ which defines the orientation since $* d f=d f \circ J$.

Remark 1. Equation (2.3) is a generalization of the Cauchy-Riemann equation: to $h: M \rightarrow \mathbb{C}$ let $f=h j: M \rightarrow \operatorname{Im} \mathbb{H}$ with $N=i$. Then (2.3) becomes

$$
* d h=i d h
$$

which is the Cauchy-Riemann equation for $h$. In this sense surface theory in $\operatorname{Im\mathbb {H}}$ can be viewed as a generalization of complex function theory to a quaternionic function theory. Holomorphic chart maps are special cases of conformal immersions.

Any $\mathbb{R}^{3}$-valued 1-form $\alpha$ on $M$ can be decomposed into its conformal and anti-conformal parts:

$$
\alpha=\alpha_{+}+\alpha_{-}, \quad * \alpha_{ \pm}= \pm N \alpha_{ \pm}
$$

where

$$
\alpha_{ \pm}=\frac{1}{2}(\alpha \pm N * \alpha)
$$

Thus, using (2.3),

$$
d * d f=d N \wedge d f=d N * d f-* d N d f=(d N N-* d N) d f=2(d N)_{+} N d f
$$

which is ImIH-valued. This means

$$
<(d N)_{+}, N d f>=0
$$

or, since $d N_{+}$is also tangential,

$$
d N_{+}=H d f
$$


for some real valued function $H: M \rightarrow \mathbb{R}$. Clearly, $H$ is the mean curvature of $f$ w.r.t. the induced metric $|d f|^{2}$. Inserting (2.5) we obtain

$$
\mathrm{d} N=H d f+\omega, \quad \omega=(d N)_{-}, \quad * \omega=-N \omega
$$

and thus

$$
d * d f=2 H N|d f|^{2} .
$$

Differentiating (2.6) we get the Codazzi equation

$$
\mathrm{d} \omega=(* d H-d H N) d f .
$$

Definition 2.1. Two conformal immersions $f, \tilde{f}: M \rightarrow \mathbb{R}^{3}$ are called spin-equivalent if there exists $\lambda: M \rightarrow \mathbb{H}_{*}$ so that

$$
d \tilde{f}=\bar{\lambda} d f \lambda
$$

We call $\tilde{f}$ a spin-transform of $f$.

Remark 2. 1. If $\pi_{1}(M)=0$ then any two conformal immersions are spin-equivalent: since $d \tilde{f}$ and $d f$ are conformal 1-forms pointwise they can be mapped into each other by a rotation and scaling. But this is exactly what (2.9) means. Notice that $\lambda$ is unique up to sign.

2. If $\pi_{1}(M) \neq 0$ then $f$ and $\tilde{f}$ are spin-equivalent if and only if they belong to the same regular homotopy class [10].

3. $\lambda$ is constant if and only if $\tilde{f}$ is obtained from $f$ by an Euclidean motion and scaling. If $|\lambda|=1$ then $\tilde{f}$ and $f$ are congruent immersions.

4. Spin-equivalence clearly is an equivalence relation.

To obtain new conformal immersions (locally) from a given reference immersion $f: M \rightarrow \mathbb{R}^{3}$ via spin-transformations we only have to solve

$$
0=d(\bar{\lambda} d f \lambda)=d \bar{\lambda} \wedge d f \lambda-\bar{\lambda} d f \wedge d \lambda=-2 \operatorname{Im}(\bar{\lambda} d f \wedge d \lambda) .
$$

But this says that $\bar{\lambda} d f \wedge d \lambda$ is real valued and hence there exists a unique real valued function $\rho: M \rightarrow \mathbb{R}$ with

$$
d f \wedge d \lambda=-\rho|d f|^{2} \lambda=\rho d f^{2} \lambda .
$$

Using (2.2), (2.3) and dividing by $d f$ we obtain the integrability equation

$$
* d \lambda+N d \lambda=\rho d f \lambda \text {. }
$$

This is worth formulating as a

Lemma 2.2. If $f, \tilde{f}: M \rightarrow \mathbb{R}^{3}$ are spin-equivalent via $d \tilde{f}=\bar{\lambda} d f \lambda$ then $\lambda: M \rightarrow \mathbb{H}_{*}$ satisfies (2.10).

Conversely, if $\pi_{1}(M)=0$ then to a given conformal immersion $f: M \rightarrow \mathbb{R}^{3}$ nowhere vanishing solutions to (2.10) yield all conformal immersions $\tilde{f}: M \rightarrow \mathbb{R}^{3}$ via spin-transformations $d \tilde{f}=\bar{\lambda} d f \lambda$.

We now relate elementary geometric data of spin-equivalent immersions.

Lemma 2.3. Let $f, \tilde{f}: M \rightarrow \mathbb{R}^{3}$ be spin-equivalent via $d \tilde{f}=\bar{\lambda} d f \lambda$. Then

1. $\tilde{N}=\lambda^{-1} N \lambda$ is the oriented normal to $\tilde{f}$,

2. $|d \tilde{f}|^{2}=|\lambda|^{4}|d f|^{2}$, 
3. $\tilde{H}=\frac{H+\rho}{|\lambda|^{2}}$ where $\rho: M \rightarrow \mathbb{R}$ is given by 2.10 .

Proof. (i) and (ii) are obvious. To see (iii) we use (2.6):

$$
\tilde{H} d \tilde{f}+\tilde{\omega}=d \tilde{N}=\frac{H}{|\lambda|^{2}} d \tilde{f}+\lambda^{-1}\left(\omega+N d \lambda \lambda^{-1}-d \lambda \lambda^{-1} N\right) \lambda,
$$

hence, computing the conformal part (2.4) of the latter and inserting (2.10), we get

$$
\begin{gathered}
\left(\lambda^{-1}\left(\omega+N d \lambda \lambda^{-1}-d \lambda \lambda^{-1} N\right) \lambda\right)_{+}=\lambda^{-1}\left(\omega+N d \lambda \lambda^{-1}-d \lambda \lambda^{-1} N\right)_{+} \lambda= \\
\lambda^{-1} \rho d f \lambda=\frac{\rho d \tilde{f}}{|\lambda|^{2}}
\end{gathered}
$$

and thus

$$
\tilde{H}=\frac{H+\rho}{|\lambda|^{2}}
$$

Corollary 2.1. Let $f, \tilde{f}: M \rightarrow \mathbb{R}^{3}$ be spin-equivalent via $d \tilde{f}=\bar{\lambda} d f \lambda$. Then the following are equivalent:

1. $\tilde{H}|d \tilde{f}|=H|d f|$

2. $d f \wedge \mathrm{d} \lambda=0$ which is the same as $* d \lambda+N d \lambda=0$.

\section{ISOTHERMIC SURFACES AND BONNET PAIRS}

We now give the local classification of all Bonnett pairs and explain how they are obtained from isothermic surfaces.

Definition 3.1. A conformal immersion $f: M \rightarrow \mathbb{R}^{3}$ is isothermic if there exists an non-zero, $\operatorname{Im} \mathbb{H}$-valued, closed and anti-conformal 1-form $\tau \in \Omega^{1}(M, \operatorname{Im} \mathbb{H})$, i.e.,

$$
\mathrm{d} \tau=0, \quad \tau \neq 0, \quad * \tau+N \tau=0 \text { which is equivalent to } d f \wedge \tau=0 .
$$

Note that this is a Moebius invariant condition.

Remark 3. 1. The classical notion of an isothermic surface is that $f: M \rightarrow \mathbb{R}^{3}$ admits conformal curvature line parameters (away from umbilic points). If $(x, y)$ are such parameters then one easily sees that the 1 -form

$$
\tau=\frac{1}{\left|f_{x}\right|^{2}}\left(f_{x} d x-f_{y} d y\right)=-f_{x}^{-1} d x+f_{y}^{-1} d y
$$

is closed and anti-conformal. Thus, classically isothermic surfaces are isothermic in our notion.

2. Since $\mathrm{d} \tau=0$ locally $\tau=d f^{*}$ and with $N^{*}=-N$ one sees that $f^{*}$ is a conformal immersion (a dual isothermic surface) away from zeros of $\tau$.

3. Examples of isothermic surfaces include quadrics, surfaces of revolution and surfaces of constant mean curvature (and their Moebius transforms). The latter case follows at once from (2.8) and (2.6): $\mathrm{d} \omega=0$ and $* \omega+N \omega=0$ so that we may put $\tau=\omega$. For minimal surfaces $(H=0)$ we get $d N=\omega$ so that the Gauss map $N$ is a dual surface.

4. Recently it has been shown [1, 3, [] that isothermic surfaces can be obtained from an infinite dimensional integrable system (soliton equation). This provides an infinte dimesnional space of examples of local isothermic surfaces. 
Definition 3.2. Two conformal immersions $f, \tilde{f}: M \rightarrow \mathbb{R}^{3}$ form a Bonnet pair if they induce the same metric $|d \tilde{f}|^{2}=|d f|^{2}$ and have the same mean curvature $\tilde{H}=H$, but are not congruent.

Finally we arrive at the local classification of all Bonnet pairs. We assume $\pi_{1}(M)=0$.

Theorem 3.1. Let $f: M \rightarrow \mathbb{R}^{3}$ be isothermic with dual surface $f^{*}: M \rightarrow \mathbb{R}^{3}$. Choose $\epsilon \in \mathbb{R}_{*}$, $a \in \operatorname{Im} \mathbb{H}$ and let $\lambda_{ \pm}= \pm \epsilon+f^{*}+a$. Then the spin transforms $f_{ \pm}: M \rightarrow \mathbb{R}^{3}$ given by $d f_{ \pm}=\overline{\lambda_{ \pm}} d f \lambda_{ \pm}$ form a Bonnet pair.

Conversely, every Bonnet pair arises from a 3-parameter family (determined up to scalings) of isothermic surfaces where the three parameters account for Euclidean rotations of the partners in the Bonnet pair w.r.t. each other.

Proof. From Definition 3.1 we have $d f \wedge d f^{*}=0$ and thus also $d f \wedge d \lambda_{ \pm}=0$, i.e., $* d \lambda_{ \pm}+N d \lambda_{ \pm}=0$, which is 2.10) with $\rho_{ \pm}=0$. Moreover, $\left|\lambda_{+}\right|=\left|\lambda_{-}\right|$so that $\left|d f_{+}\right|^{2}=\left|d f_{-}\right|^{2}$ and $H_{+}=H_{-}$. Congruence of $f_{+}$and $f_{-}$means that $\lambda_{+}^{-1} \lambda_{-}$is constant which would imply that $f^{*}$ is constant. Hence $f_{ \pm}$form a Bonnet pair.

To show the converse assume that we have a Bonnet pair $f_{ \pm}$. Then

$$
d f_{+}=\bar{\lambda} d f_{-} \lambda
$$

for some $\lambda: M \rightarrow \mathbb{H}$. But $f_{+}$and $f_{-}$induce the same metric and same mean curvature so that $|\lambda|=1$ and by Corollary $2.1 d f_{-} \wedge \mathrm{d} \lambda=0$. Solving for $f^{*}$ in terms of $\lambda_{+}^{-1} \lambda_{-}$in the first part of the Theorem (with $a=0$ ) suggests to define

$$
f^{*}=(\lambda-1)^{-1}+1 / 2 .
$$

To insure that $\lambda-1$ vanishes nowhere we may choose to multiply $\lambda$ on the right by a unit quaternion (which induces a rotation of the partners of the Bonnet pair w.r.t. each other). Since $|\lambda|=1$ one easily checks that $f^{*}$ is purely imaginary. Putting

$$
d f=\overline{(\lambda-1)} d f_{-}(\lambda-1)
$$

we see that $d f \wedge d f^{*}=0$ so that $f: M \rightarrow \mathbb{R}^{3}$ is isothermic with dual surface $f^{*}$. Now one easily checks that $f_{ \pm}$are spin transforms of $f$ via $\lambda_{ \pm}= \pm 1 / 2+f^{*}$.

Remark 4. 1. Choices of real $\epsilon$ and imaginary quaternionic $a$ give a 4-parameter family of Bonnet pairs to each isothermic surface. For $\epsilon=0$ the pair degenerates to a 3-paramter family of isothermic surfaces $d \tilde{f}=\overline{\left(f^{*}+a\right)} d f\left(f^{*}+a\right)$. Compactifying this $\mathbb{R}^{4}$ to $S^{4}$ adds $f$ (up to congruence) at infinity. Thus the above construction provides an $S^{4}$-worth of Bonnet pairs with an equatorial $S^{3}$ of isothermic surfaces.

2. It is known that there exists at most two compact embedded surfaces in $\mathbb{R}^{3}$ with prescribed mean curvature and metric [9]. Understanding the period problem in our local classification could provide an affirmative solution to the question of whether a given compact embedded surface allows a Bonnet partner.

\section{REFERENCES}

1. F. Burstall, U. Hertrich-Jeromin, F. Pedit and U. Pinkall. Curved flats and Isothermic surfaces. To appear in Math. Z.

2. A. Bobenko and U. Eitner. Bonnet surfaces and Painleve equations. SFB preprint 151, 1994.

3. A. Bobenko and U. Pinkall. Discrete isothermic surfaces. J. reine angew. Math. 457, (1996), 187-208. 
4. E. Cartan. Sur les couples de surfaces applicables avec conservation des courbures principales. Bull. Sc. Math. 66, (1942).

5. S.S. Chern. Deformations of surfaces preserving principal curvatures. Differential Geometry and Complex Analysis: a volume dedicated to the memory of H.E. Rauch. Springer Verlag 1985, 155-163.

6. O. Bonnet. Memoire sur la theorie des surfaces applicables. J. Ec. Polyt. 66, (1842).

7. U. Hertrich-Jeromin and F. Pedit. Remarks on the Darboux transform of isothermic surfaces. GANG preprint 1996.

8. G. Kamberov. Recovering the shape of a surface from the mean curvature. Dynamical Systems and Differential equations. M. Dekker 1996. Submitted.

9. B. Lawson and R. Tribuzy. On the mean curvature function for compact surfaces. J. Diff. Geom. 3, (1987).

10. U. Pinkall. Regular homotopy classes of immersed surfaces. Topology 24, (1985), 412-434.

Department of Mathematics, University of Massachusetts, Amherst, MA 01003

E-mail address, George Kamberov: george@gang.umass.edu

Department of Mathematics, University of Massachusetts, Amherst, MA 01003

E-mail address, Franz Pedit: franz@gang.umass.edu

Fachbereich Mathematik, MA 8-3, Technische Universität Berlin, Strasse des $17 . \quad$ Juni 136,10622 Berlin, Germany

E-mail address, Ulrich Pinkall: pinkall@sfb288.math.tu-berlin.de 